\title{
Impaired branched chain amino acid metabolism alters feeding behavior and increases orexigenic neuropeptide expression in the hypothalamus
}

\author{
Megan N Purpera, Li Shen, Marzieh Taghavi ${ }^{1}$, Heike Münzberg, Roy J Martin, Susan M Hutson ${ }^{1}$ \\ and Christopher D Morrison
}

Pennington Biomedical Research Center, Baton Rouge, Louisiana 70808, USA

${ }^{1}$ Department of Human Nutrition, Foods and Exercise, Virginia Polytechnic Institute, Blacksburg, Virginia 24061, USA

(Correspondence should be addressed to C D Morrison; Email: morriscd@pbrc.edu)

\begin{abstract}
Elevation of dietary or brain leucine appears to suppress food intake via a mechanism involving mechanistic target of rapamycin, AMPK, and/or branched chain amino acid (BCAA) metabolism. Mice bearing a deletion of mitochondrial branched chain aminotransferase (BCATm), which is expressed in peripheral tissues (muscle) and brain glia, exhibit marked increases in circulating BCAAs. Here, we test whether this increase alters feeding behavior and brain neuropeptide expression. Circulating and brain levels of BCAAs were increased two- to four-fold in BCATm-deficient mice (KO). KO mice weighed less than controls $(25.9$ vs $20.4 \mathrm{~g}, \quad P<0.01)$, but absolute food intake was relatively unchanged. In contrast to wild-type mice, $\mathrm{KO}$ mice preferred a low-BCAA diet to a control diet $(P<0 \cdot 05)$ but exhibited no change in preference for
\end{abstract}

low- vs high-protein (HP) diets. KO mice also exhibited low leptin levels and increased hypothalamic Npy and Agrp mRNA. Normalization of circulating leptin levels had no effect on either food preference or the increased Npy and Agrp mRNA expression. If BCAAs act as signals of protein status, one would expect reduced food intake, avoidance of dietary protein, and reduction in neuropeptide expression in BCATm-KO mice. Instead, these mice exhibit an increased expression of orexigenic neuropeptides and an avoidance of BCAAs but not HP. These data thus suggest that either BCAAs do not act as physiological signals of protein status or the loss of BCAA metabolism within brain glia impairs the detection of protein balance.

Journal of Endocrinology (2012) 212, 85-94

\section{Introduction}

An adequate supply of protein is necessary for life, and a number of observations support the existence of regulatory systems that assess endogenous protein/amino acid demand and alter food intake to meet this demand (Peters \& Harper 1984, Hannah et al. 1990, White et al. 1994, Du et al. 2000, Jean et al. 2001, Westerterp-Plantenga 2003, Anderson \& Moore 2004, Lacroix et al. 2004, Tome 2004). This regulatory drive to consume adequate protein can be extremely powerful, resulting in protein intake being regulated as a higher priority than energy intake (Sorensen et al. 2008, Brooks et al. 2010). Yet, to date, very little is known about the mechanisms underlying 'protein balance' and its relation with energy balance.

To achieve this regulation, it seems likely that the brain responds to variations in circulating signals that reflect protein balance. To date, some evidence supports alterations in circulating hormones (Batterham et al. 2006), while other works suggest that individual amino acids may act locally within the brain (Panksepp \& Booth 1971, Sandoval et al. 2008). For instance, the branched chain amino acid (BCAA) leucine suppresses food intake when locally administered into the brain (Cota et al. 2006, Morrison et al. 2007, Ropelle et al. 2008, Blouet et al. 2009), and supplementing the diet with excess leucine or BCAAs also suppresses food intake (Ropelle et al. 2008, Newgard et al. 2009). Together, these data suggest that BCAA, and particularly leucine, may be uniquely suited to serve as signals of protein balance.

To date, the majority of experiments have focused on mechanistic target of rapamycin (MTOR; also known as mammalian target of rapamycin, $\mathrm{mTOR}$ ) as a probable mediator of brain leucine signaling (Cota et al. 2006, Morrison et al. 2007, Ropelle et al. 2008). However, Blouet et al. (2009) demonstrated that local injection of downstream products of BCAA metabolism ( $\boldsymbol{\alpha}$-ketoisocaproic acid or $\alpha$-ketoisovaleric acid), or pharmacologic manipulation of BCAA metabolism, also altered food intake. As such, it is possible that BCAA metabolism may also produce signals relevant to food intake or protein detection. 
To test the effect of altered BCAA metabolism on feeding behavior and hypothalamic function, we focused on mice bearing a whole-body deletion of the mitochondrial form of branched chain aminotransferase (BCATm; She et al. 2007). Because these mice exhibit marked increases in circulating BCAAs, they provide a novel model system in which to test whether increased circulating BCAAs represent a signal of protein excess. We hypothesized that BCATm-KO mice would exhibit a phenotype consistent with excess protein, including reduced food intake, reduced orexigenic neuropeptide gene expression, and avoidance of high-protein (HP) diets.

\section{Materials and Methods}

\section{Animals}

BCATm-deficient mice (KO mice) were developed as described previously by She et al. (2007), who also described the general phenotype of increased circulating BCAAs, leanness, and increased oxygen consumption. All studies used male KO mice or wild-type (WT) littermates generated at Virginia Tech (Experiment 1) or from breeders subsequently sent to Pennington Biomedical Research Center (Experiments 2 and 3). Mice were group housed (two to four per cage) in shoebox cages under a $12 \mathrm{~h}$ light:12 h darkness cycle and were fed standard chow made available ad libitum unless otherwise noted. All experiments were approved by the Institutional Animal Care and Use Committee of Pennington Biomedical Research Center.

\section{Experiment 1: brain amino acids and orexigenic neuropeptides in $\mathrm{KO}$ mice}

To identify baseline differences in plasma and brain amino acids and hypothalamic neuropeptide expression, 20-week-old male homozygous BCATm-deficient mice (KO; $n=5$ ) or WT littermates $(n=10)$ were rapidly killed, trunk blood was collected, and brains were isolated. Samples of mediobasal hypothalamus containing principally arcuate nucleus were isolated for RNA extraction, while forebrains were collected to assess brain amino acid concentrations via HPLC (see below).

Experiment 2: effects of leptin replacement on feeding behavior, body adiposity, and hypothalamic neuropeptides in KO mice

To determine whether normalization of circulating leptin levels would normalize hypothalamic neuropeptides and food intake, 8-week-old male BCATm-KO mice and WT littermates were adapted to a two-choice diet paradigm in which mice were allowed to self-select between a control diet and a diet containing BCAAs at only $2 \cdot 5 \%$ of the control (BCAA-low). Mice were group housed and acclimated to the two-choice paradigm for 2 weeks and were then singlehoused prior to implantation with s.c. osmotic minipumps (Alzet Minipumps, Durect, Cupertino, CA, USA) delivering either PBS, $3 \mu \mathrm{g} /$ day leptin, or $10 \mu \mathrm{g}$ /day leptin $(n=9-10$ per group) (White et al. 2009). Leptin was procured from the National Hormone Pituitary Program (Dr A F Parlow). Food intake and body weight were measured daily, and after 6 days of infusion, mice were rapidly killed, retroperitoneal and epididymal fat pads were collected and weighed, and hypothalamus was isolated for RNA extraction.

To test whether $\mathrm{KO}$ mice also exhibited an altered preference for low-protein (LP)- vs HP diet, WT $(n=9)$ and $\mathrm{KO}(n=13)$ mice were offered a choice between isocaloric diets providing casein protein at $10 \%$ of energy (LP) or $35 \%$ of energy (HP; Table 1). Food intake was measured daily for 10 days, but mice were otherwise not manipulated.

Experiment 3: effects of acute, high-dose leptin treatment on hypothalamic neuropeptides in control and BCATm-KO mice

To determine whether KO mice were unresponsive to leptindependent regulation of mRNA expression, BCATm-KO mice and WT littermates received i.p. injections of leptin in the fed or fasted state ( $n=8$ per group). Mice received an i.p. injection of either leptin ( $5 \mu \mathrm{g} / \mathrm{g}$ body weight) or saline, at the beginning of the fast (Time 0) and then after 12 and $24 \mathrm{~h}$. Four hours after the final injection, mice were killed and hypothalami were isolated for RNA extraction.

\section{Amino acid analysis}

Brain and plasma amino acids were measured using fluorometric HPLC via methods described previously (Wu \& Knabe 1994), except that methanol extraction was used for brain tissue rather than acid precipitation. The o-phthaldialdehyde amino acid derivatives were separated by gradient elution using a Supelcosil LC-18 column $(15 \mathrm{~cm} \times 4.6 \mathrm{~mm}$, $3 \mu \mathrm{m}$, Sigma).

\section{Hormone analysis}

Trunk blood was collected and allowed to clot at $4{ }^{\circ} \mathrm{C}$ overnight, centrifuged at $3000 \mathrm{~g}$ for $30 \mathrm{~min}$, and serum collected and stored at $-80^{\circ} \mathrm{C}$. Serum levels of leptin were measured using RIA kit (Millipore, St Charles, MO, USA), according to the manufacturer's instructions.

\section{$R N A$ extraction and real-time PCR}

RNA extraction and real-time PCR were conducted as described previously (Morrison et al. 2007, White et al. 2010). Total RNA was extracted from mediobasal hypothalamus using Tri-Reagent (Molecular Research Center, Cincinnati, $\mathrm{OH}$, USA), according to the manufacturer's instructions. Confirmation of intact $18 S$ and $28 S$ RNA bands was achieved by ethidium bromide staining after electrophoresis of samples in $1 \%$ agarose gels. Samples were quantified by spectrophotometry, then mRNA was reverse transcribed 
Table 1 Experimental diets

\begin{tabular}{|c|c|c|c|c|}
\hline Ingredient (g) & Control & Low BCAA & Low protein & High protein \\
\hline Casein & 0 & 0 & 100 & 350 \\
\hline Corn starch & 300 & 300 & $440 \cdot 3$ & $291 \cdot 7$ \\
\hline Maltodextrin & 125 & 125 & 150 & 75 \\
\hline Sucrose & 250 & 250 & $107 \cdot 077$ & $107 \cdot 077$ \\
\hline Cellulose & 50 & 50 & 50 & 50 \\
\hline Soybean oil & 50 & 50 & 25 & 25 \\
\hline Lard & 0 & 0 & 75 & 75 \\
\hline Mineral Mix S10001 & 35 & 35 & 0 & 0 \\
\hline Mineral Mix S10022C & 0 & 0 & $3 \cdot 5$ & $3 \cdot 5$ \\
\hline Sodium bicarbonate & $7 \cdot 5$ & $7 \cdot 5$ & 0 & 0 \\
\hline Vitamin mix & 10 & 10 & 10 & 10 \\
\hline Choline bitartrate & 2 & 2 & $2 \cdot 5$ & 0 \\
\hline Diammonium citrate & 0 & 24 & 0 & 0 \\
\hline Calcium carbonate & 0 & 0 & 10 & $12 \cdot 38$ \\
\hline Potassium citrate & 0 & 0 & $2 \cdot 48$ & $6 \cdot 58$ \\
\hline Potassium phosphate & 0 & 0 & $6 \cdot 86$ & $1 \cdot 6$ \\
\hline Sodium chloride & 0 & 0 & $2 \cdot 59$ & $2 \cdot 59$ \\
\hline L-arginine & $8 \cdot 27$ & $8 \cdot 27$ & & \\
\hline L-histidine- $\mathrm{HCl}-\mathrm{H}_{2} \mathrm{O}$ & 6 & 6 & & \\
\hline L-isoleucine & 8 & $0 \cdot 205$ & & \\
\hline L-leucine & 12 & $0 \cdot 278$ & & \\
\hline L-lysine-HCl & 14 & 14 & & \\
\hline DL-methionine & 6 & 6 & & \\
\hline L-phenylalanine & 8 & 8 & & \\
\hline L-threonine & 8 & 8 & & \\
\hline L-tryptophan & 2 & 2 & & \\
\hline L-valine & 8 & $0 \cdot 205$ & & \\
\hline L-alanine & 10 & 10 & & \\
\hline L-asparagine- $\mathrm{H}_{2} \mathrm{O}$ & 10 & 10 & & \\
\hline L-cystine & 4 & 4 & $1 \cdot 5$ & $5 \cdot 25$ \\
\hline L-glutamic acid & 10 & 10 & & \\
\hline L-glutamine & 10 & 10 & & \\
\hline Glycine & 10 & 10 & & \\
\hline L-proline & 10 & 10 & & \\
\hline L-serine & 10 & 10 & & \\
\hline L-tyrosine & 4 & 4 & & \\
\hline Total & $987 \cdot 8$ & $984 \cdot 5$ & 990 & 1018 \\
\hline $\mathrm{kcal} / \mathrm{g}$ & $3 \cdot 87$ & $3 \cdot 77$ & $4 \cdot 13$ & $4 \cdot 02$ \\
\hline
\end{tabular}

into cDNA, and mRNA expression determined using the SYBR green methodology in optical 384-well plates in an ABI PRISM 7900 sequence detector (Applied Biosystems, Branchburg, NJ, USA). All expression data were normalized to cyclophilin mRNA levels.

\section{Statistical analysis}

Data were analyzed using the SAS software package (SAS V9, SAS Institute, Cary, NC, USA) using a two-tailed $t$-test or ANOVA using the general linear model procedure. When experiment wide tests were significant, post hoc comparisons were made using the LSMEANS statement with the PDIFF option and thus represent least significant differences tests for pre-planned comparisons. For real-time PCR, expression levels were normalized to cyclophilin prior to analysis. All data are expressed as mean \pm s.E.M., with a probability value of $0 \cdot 05$ considered statistically significant.

\section{Results}

Experiment 1: impact of BCATm deletion on brain amino acids and orexigenic neuropeptides

Similar to previous experiments (She et al. 2007), BCATm$\mathrm{KO}$ mice exhibited a marked increase in circulating BCAA levels $(P<0 \cdot 01$; Fig. $1 \mathrm{~A})$, with plasma leucine, isoleucine, and valine concentrations increased by four-, eight-, and sevenfold respectively. Similarly, brain BCAA levels were increased by two-, five-, and three-fold respectively $(P<0 \cdot 01$; Fig. $1 \mathrm{~B})$. This ratio of blood to brain levels of BCAAs is consistent with the kinetic properties of the system $\mathrm{L}$ amino acid transporters (LAT1) expressed in tissues forming the blood-brain barrier (Kanai et al. 1998). The increased BCAA concentrations had a variable effect on brain levels of non-BCAAs (Table 2). For example, Glu and Gln concentrations were statistically higher in the BCATm-KOs compared with WT controls and GABA 


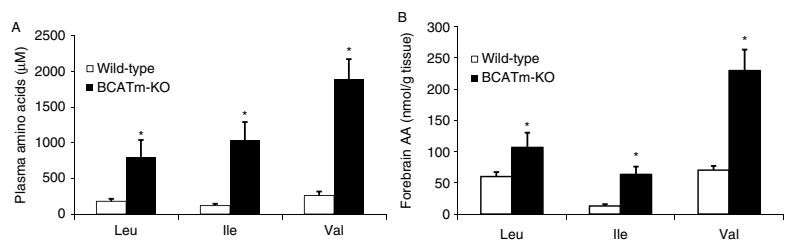

Figure 1 Plasma and brain BCAAs in $\mathrm{KO}$ and wild-type (WT) mice. Plasma (A) and brain (B) BCAAs were measured in BCATm-KO mice $(n=5)$ and WT littermates ( $n=10 ; 20$ weeks of age) using fluorometric HPLC methods. For brain measures, forebrains were utilized, as hypothalami were used to assess neuropeptide expression. Levels of brain and plasma BCAA were increased in KO compared with control mice. ${ }^{*} P<0 \cdot 05$ compared to WT.

was unchanged. Amino acids that are precursors of the monoamine neurotransmitters and/or substrates for LAT1 were either unchanged (Phe, Trp, and Met) or higher (Tyr). These data do not support the possibility that elevations in BCAAs interfered with brain uptake of other large, neutral amino acids (Tews et al. 1979).

Agrp mRNA levels were significantly increased within BCATm-deficient mice as compared with controls $(P<0 \cdot 05$; Fig. 2). Npy mRNA levels were roughly doubled, but this difference did not reach statistical significance $(P=0 \cdot 14)$. Pomc mRNA expression was also measured, but the results were inconsistent between experiments and as such inconclusive (data not shown). Interestingly, changes in hypothalamic Npy and Agrp mRNA expression are consistent with the profile of animals on LP diets (White et al. 1994, Morrison et al. 2007).

\section{Experiment 2: role of hypoleptinemia in the phenotype of $B C A T$-deficient mice}

Two weeks prior to minipump implantation, mice were transferred to a 2-choice diet paradigm offering the choice between a control diet and a diet very low in BCAAs $(2 \cdot 5 \%$ BCAAs compared to control; Table 1). KO mice were smaller than WT mice $(P<0 \cdot 001$; Fig. $3 \mathrm{~A})$, and this reduction in body weight was highly consistent with previous work in these mice (She et al. 2007). Baseline food intake (prior to minipump) was not different between KO and WT mice on an absolute basis (Fig. 3C), but when food intake was adjusted for body weight, KO mice consumed more food than controls $(P<0 \cdot 001$; Fig. 3D). While differences in absolute intake were relatively subtle, KO and WT mice exhibited marked differences in their preference between the control and low-BCAA diets $(P<0 \cdot 001 ;$ Fig. 3B). While WT mice significantly preferred the control diet, $\mathrm{KO}$ mice preferred the diet with very low BCAAs. KO mice therefore appear to avoid dietary BCAAs, presumably in response to their excess endogenous BCAAs.

To test whether the elevation of circulating BCAAs represents a signal of excess protein, a separate group of BCATm-deficient mice and control mice were maintained on more generic HP (35\% casein protein, Table 1) and LP diets $(10 \%$ casein protein). BCATm-KO mice exhibited a subtle decrease in absolute food intake compared with controls (Fig. 4C), which was accounted for by adjusting to body weight (Fig. 4D). Despite their elevation of circulating BCAAs, KO mice did not exhibit altered selection between the LP and HP diets (Fig. 4B). Thus, the specific avoidance of BCAAs in the KO mice did not translate into a more generalized avoidance of protein.

Following this 2-week dietary acclimation period, male KO mice and their WT littermates were implanted with osmotic minipumps designed to a) return plasma leptin levels to normal following administration of leptin at a low dose $(3 \mu \mathrm{g} /$ day $)$ and $\mathrm{b})$ increase leptin above normal following administration of leptin at a higher dose $(10 \mu \mathrm{g} /$ day $)$. Circulating leptin levels were reduced in $\mathrm{KO}$ mice $(P<0 \cdot 05$; Fig. 5), although the difference was not as large as previously reported (She et al. 2007). Infusion of $3 \mu \mathrm{g} /$ day leptin normalized circulating leptin to physiological levels in $\mathrm{KO}$ mice and produced a small but significant increase in the WT mice. In contrast, the higher $10 \mu \mathrm{g} /$ day dose produced a marked increase in both KO and WT mice. Leptin treatment had little effect on body weight gain over the 6 days of infusion, as only $\mathrm{KO}$ mice receiving the highest dose exhibited a subtle decrease in weight $(P=0.054$; data not shown). Leptin significantly suppressed total daily food intake in both genotypes $(P<0 \cdot 05$; Fig. $6 \mathrm{~A})$, such that there was a significant main effect of leptin treatment $(P<0 \cdot 001)$, but no significant treatment by genotype interaction $(P=0.53)$. Although it suppressed total intake, leptin treatment had no effect on the preference ratio between the control and lowBCAA-diets in either genotype (Fig. 6B) such that KO mice

Table 2 Forebrain amino acids $(\mu \mathrm{M})$ in BCATm-KO mice compared to wild-type littermates

\begin{tabular}{|c|c|c|c|}
\hline Amino acid & Wild-type & ВСАTm-KO & $\boldsymbol{P}$ \\
\hline TYR & $37 \pm 9$ & $127 \pm 12$ & $0 \cdot 001 *$ \\
\hline ORN & $314 \pm 29$ & $460 \pm 1$ & $0 \cdot 001 *$ \\
\hline GLN & $4116 \pm 497$ & $6388 \pm 595$ & $0 \cdot 002 *$ \\
\hline ASN & $144 \pm 8$ & $187 \pm 14$ & $0 \cdot 01^{*}$ \\
\hline THR & $452 \pm 54$ & $601 \pm 73$ & $0 \cdot 02 *$ \\
\hline ALA & $823 \pm 193$ & $724 \pm 31$ & 0•02* \\
\hline LYS & $81 \pm 5$ & $99 \pm 12$ & $0 \cdot 02 *$ \\
\hline GLU & $9509 \pm 603$ & $10532 \pm 563$ & $0 \cdot 03^{*}$ \\
\hline SER & $840 \pm 50$ & $916 \pm 61$ & $0 \cdot 03^{*}$ \\
\hline ARG & $287 \pm 29$ & $365 \pm 32$ & $0 \cdot 07$ \\
\hline ASP & $1993 \pm 214$ & $2264 \pm 199$ & $0 \cdot 13$ \\
\hline MET & $75 \pm 6$ & $71 \pm 18$ & $0 \cdot 21$ \\
\hline TRP & $22 \pm 4 \cdot 8$ & $14 \pm 6 \cdot 6$ & $0 \cdot 22$ \\
\hline CIT & $16 \pm 1 \cdot 1$ & $22 \pm 1 \cdot 5$ & $0 \cdot 24$ \\
\hline GLY & $983 \pm 95$ & $1178 \pm 83$ & $0 \cdot 5$ \\
\hline TAU & $9357 \pm 225$ & $9125 \pm 125$ & 0.67 \\
\hline PHE & $51 \pm 3 \cdot 1$ & $50 \pm 11$ & 0.93 \\
\hline GABA & $2859 \pm 280$ & $3020 \pm 126$ & 0.97 \\
\hline
\end{tabular}

$* P<0 \cdot 05$ 


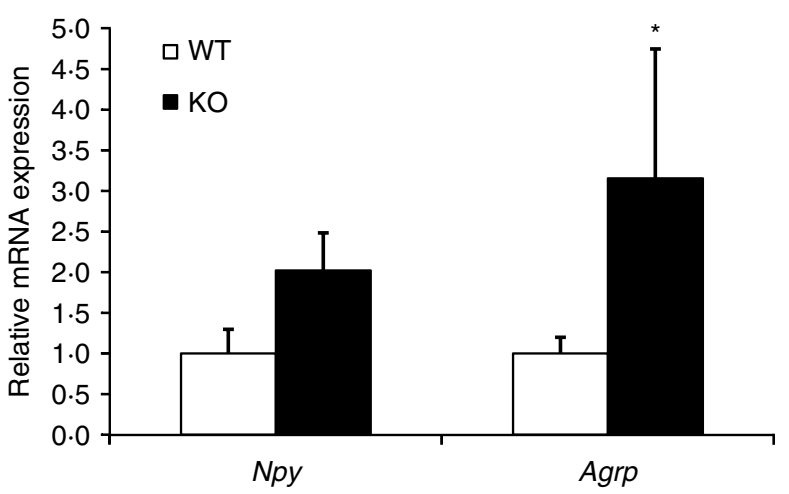

Figure 2 Hypothalamic Npy and Agrp mRNA expression in $\mathrm{KO}$ and wild-type (WT) mice. Total RNA was extracted from hypothalamus from 20-week-old BCATm-KO mice $(n=5)$ and WT littermates $(n=10)$, and expression of Npy and Agrp mRNA was measured via real-time PCR. Npy mRNA levels were numerically but not statistically increased, while Agrp mRNA expression was significantly increased in $\mathrm{KO}$ mice. ${ }^{*} P<0 \cdot 05$ compared to WT.

continued to exhibit a marked shift in preference toward the low-BCAA diet despite normalization of circulating leptin.

KO mice were lean compared with WT controls, exhibiting significantly lower retroperitoneal and epididymal fat pad weights $(P<0 \cdot 01$; Fig. 7). While leptin had little effect on adiposity in control mice, the KO mice exhibited significant decreases in body fat, particularly at the high dose $(P<0 \cdot 05$; Fig. 7$)$.

The primary goal of Experiment 2 was to determine whether normalization of circulating leptin levels would reduce hypothalamic Npy and Agrp mRNA expression in BCATm-KO mice. Similar to the results from Experiment 1, a significant increase in $N p y$ and Agrp levels was detected within $\mathrm{KO}$ mice $(P<0 \cdot 01$; Fig. $8 \mathrm{~A})$. However, increasing circulating leptin had no effect on either $N p y$ (Fig. 8B) or Agrp (Fig. 8C) mRNA expression within KO mice. These data suggest that the increase in $N p y$ and Agrp mRNA levels in BCATm-KO mice cannot be explained by reduced circulating leptin.

Experiment 3: effects of acute, high-dose leptin treatment on hypothalamic neuropeptides in control and BCATm-KO mice

It is possible that hypothalamic NPY/AgRP neurons are rendered resistant to the effects of leptin in BCATm-KO mice and that this loss of sensitivity explains the lack of effect of leptin on Npy and Agrp mRNA levels in these mice. To assess this possibility, a separate group of BCATm-KO mice was treated with acute doses of leptin every $12 \mathrm{~h}$ during a $28 \mathrm{~h}$ fast, which is a paradigm more typically used to assess the effects of leptin on hypothalamic gene expression (Baskin et al. 1999, Mizuno \& Mobbs 1999, Korner et al. 2001, Morrison et al. 2005). While leptin had no effect on either Npy or Agrp in the fed state, fasting increased both $N p y$ and Agrp mRNA expression $(P<0.05$; Fig. 9), and injection with leptin blocked this fasting induced increase.

\section{Discussion}

Nutrients such as glucose, fatty acids, and amino acids can act locally within the brain to regulate neuronal function, food intake, and body weight (Sandoval et al. 2008, Blouet \& Schwartz 2010, Moran 2010). Dietary protein content or physiological protein demand influences food intake and/or food selection (White et al. 1994, Westerterp-Plantenga 2003, Anderson \& Moore 2004, Tome 2004), as well as energy expenditure (Zhang et al. 2007, Smeets et al. 2008, Westerterp-Plantenga et al. 2009). Collectively, these observations suggest that regulatory systems detect variations in protein balance and regulate food intake, food selection, and metabolism accordingly (Sorensen et al. 2008, Brooks et al. 2010). Significant emphasis has recently been placed on the BCAA leucine, based on its ability to suppress food intake when administered directly into the brain or following supplementation in the diet (Cota et al. 2006, Morrison et al. 2007, Ropelle et al. 2008, Blouet et al. 2009, Newgard et al. 2009). Yet, some uncertainty exists as to the physiological relevance and specific signaling mechanism(s) underlying brain leucine signaling. Several lines of evidence indicate that MTOR signaling is an important mediator, as central leucine activates MTOR, and rapamycin pretreatment blocks the effects of leucine (Cota et al. 2006, Ropelle et al. 2008, Blouet et al. 2009). However, administration of a downstream
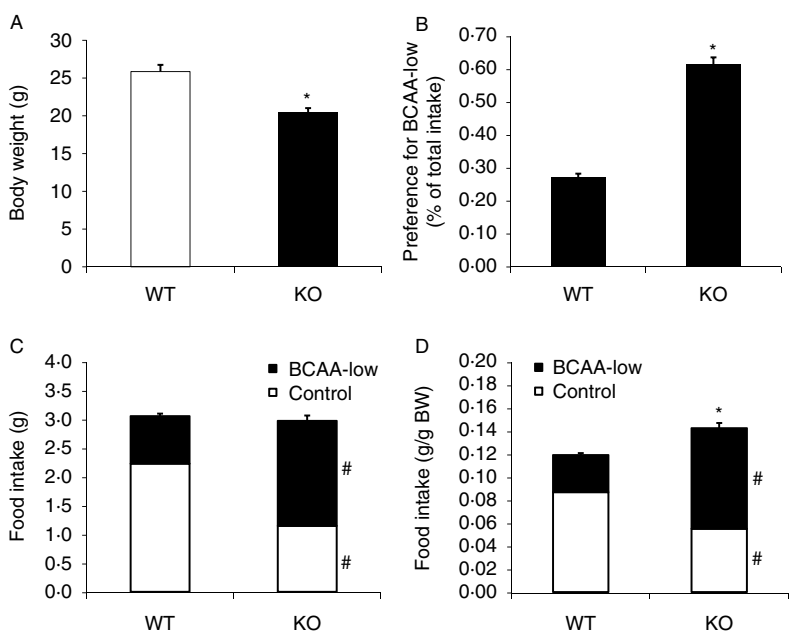

Figure 3 Body weight, food intake, and food preference in BCATmKO mice. Eight-week-old BCATm-KO mice $(n=22)$ and wild-type (WT) controls $(n=30)$ were group housed (two to three per cage) and offered the choice between a control diet and a diet containing only $2.5 \%$ of control levels of BCAAs (BCAA-low). Body weights (A) and per cage food intake ( $n=9-10$ per group) were measured, with food intake being expressed as both total intake (C) and intake adjusted for body weight (D). Food intake is also presented as a preference ratio for the low-BCAA diet (BCAA-low divided by total intake, B). KO mice were lighter than WT mice, and food intake relative to body weight was significantly increased. Most strikingly, KO mice exhibit a marked shift in preference toward the low-BCAA diet $* P<0 \cdot 05$ compared to WT. ${ }^{*} P<0.05$ for individual diet choices compared with WT. 

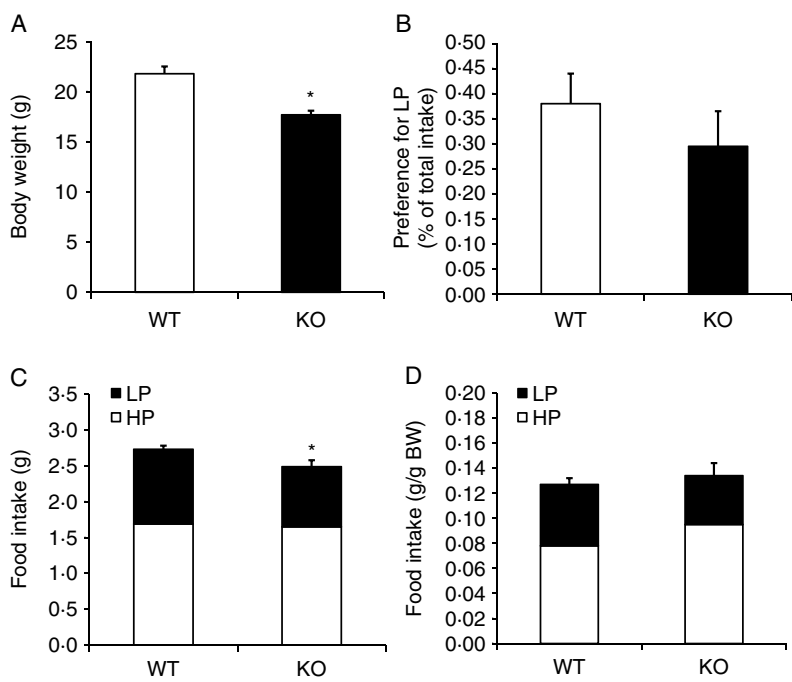

Figure 4 Food intake and selection between low-protein (LP) and high-protein (HP) diets in BCATm-KO mice. Eight-week-old BCATm-KO $(n=9)$ and wild-type (WT) littermates $(n=13)$ were single-housed and offered a choice between HP (35\% protein energy) and LP (10\% protein energy) diets. Body weight (A), preference ratio for $\mathrm{LP}$ diet $(\mathrm{B})$, total food intake $(\mathrm{C})$, and body weight adjusted food intake (D) were measured for 10 days. KO mice weighed less and consumed less total food than WT mice, but there was no difference between the genotypes regarding preference for LP vs HP diet. ${ }^{*} P<0 \cdot 05$ compared to WT.

product of BCAA metabolism or pharmacological activation of BCAA oxidation was also shown to reduce food intake (Blouet et al. 2009), suggesting that the metabolism of BCAAs might generate regulatory signals.

Considering this evidence, we utilized BCATm-deficient mice as a unique model in which circulating BCAAs are markedly increased. BCATm serves as the initial step in the metabolism of BCAAs. Two individual isozymes of BCAT exist: BCATm, which is expressed in most peripheral tissues and brain glia, and BCATc, which is expressed almost exclusively within neurons (Hutson et al. 1992, Sweatt et al. 2004a,b). Because BCATm-deficient mice are unable to metabolize BCAAs within peripheral tissues, particularly muscle, they exhibit a marked increase in circulating BCAAs (She et al. 2007). Based on the previous data (Cota et al. 2006, Morrison et al. 2007, Ropelle et al. 2008, Blouet et al. 2009), one would anticipate that this elevation of circulating BCAAs would signal protein excess, resulting in a decrease in food intake and avoidance of dietary protein. In addition, because our previous work indicates that animals on a LP diet exhibit hyperphagia and increased Npy and Agrp mRNA expression (White et al. 1994, Morrison et al. 2007), our prediction was that the increased levels of BCAAs within BCATm-KO mice would act to suppress food intake and Npy and Agrp mRNA expression. Yet, the results are inconsistent with this hypothesis, as BCATm mice do not show a clear suppression of food intake, and Npy and Agrp mRNA expression was actually increased in these mice. It should be noted that while Agrp mRNA expression was increased in both experiments, $N p y$ was only significantly increased in Experiment 2. This lack of statistical significance in Experiment 1 is likely due to the low animal numbers and reduced power in this initial experiment because the pattern of expression was similar. Finally, we also measured Pomc expression in these samples, but the results were highly inconsistent across experiments. As such, the role of Pomc in contributing to the phenotype in these mice remains unclear.

At least two possible explanations exist for observed changes in hypothalamic neuropeptide expression: the first possibility is that BCAAs have no effect on hypothalamic neuropeptides in vivo and that the increases in Npy and Agrp are driven by some secondary effect (i.e. reduced circulating leptin, see below). The second possibility is that, while neuronal BCAA metabolism is intact, the loss of glia BCAA metabolism in some fashion impairs brain amino acid sensing, resulting in the increase in Npy and Agrp despite the increase in BCAAs. Considering that the observed increases in $N p y$ and Agrp are fully consistent with the changes that we observe in animals on a LP diet, and previous in vivo and in vitro experimentation suggesting that amino acids may regulate these neuropeptides, we propose that altered glial BCAA metabolism contributes at least partly to the altered neuropeptide expression in these mice. However, early work in BCATm-KO indicated very low levels of circulating leptin (She et al. 2007), which could also contribute to the observed increases in Npy and Agrp. We therefore wanted to rule out the possibility that low leptin levels contribute to the phenotype of these mice. To test this question, we chronically infused BCATm-KO mice and control littermates with two

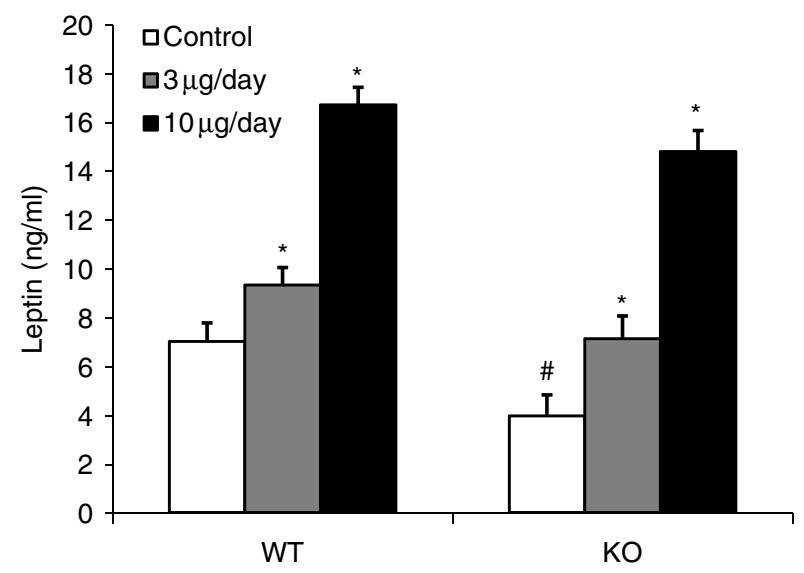

Figure 5 Leptin replacement in BCATm-KO mice. Ten-week-old BCATm-KO or wild-type mice were implanted with s.c. osmotic minipumps delivering saline, $3 \mu \mathrm{g}$ /day leptin, or $10 \mu \mathrm{g} /$ day leptin (nine to ten mice per group). Infusion lasted for 6 days, and on day 6 trunk blood leptin levels were measured via RIA. Infusion of serum leptin increased leptin at both doses in both genotypes. BCATm-KO mice receiving saline exhibited reduced circulating leptin levels, and infusion of the low dose served to fully normalize leptin in these $\mathrm{KO}$ animals. ${ }^{*} P<0.05$ compared to respective saline-infused group. ${ }^{\sharp} P<0 \cdot 05$ for WT-control vs KO-control. 

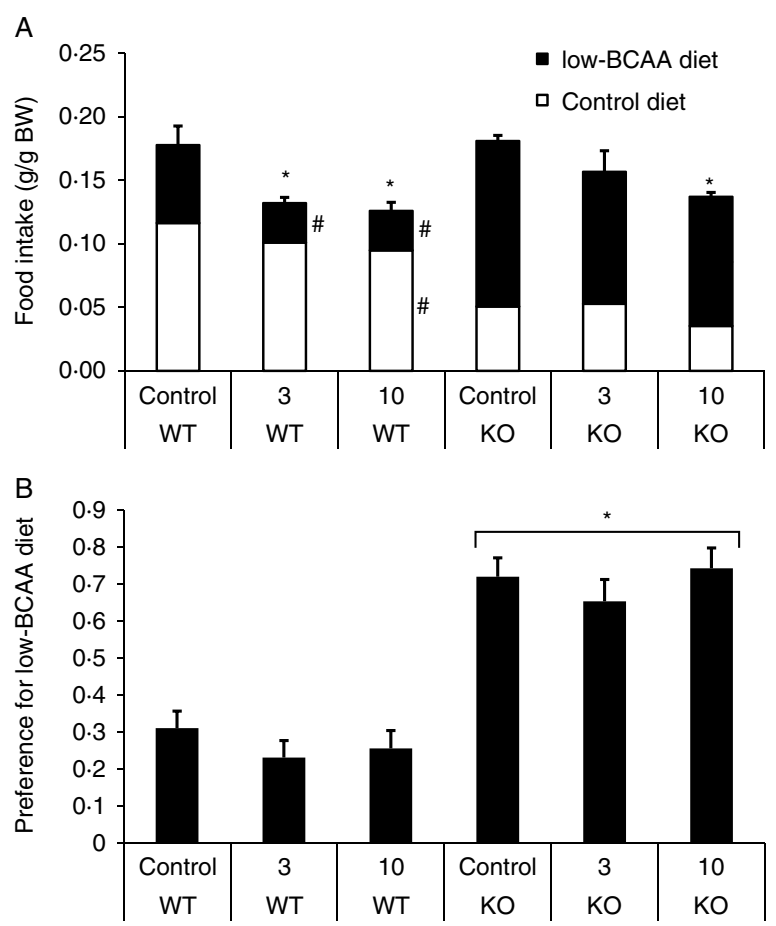

Figure 6 Food intake and food preference in BCATm-KO mice infused with leptin. BCATm-KO or wild-type mice were implanted with s.c. osmotic minipumps delivering saline, $3 \mu \mathrm{g} /$ day leptin, or $10 \mu \mathrm{g}$ /day leptin. Mice were offered the choice between a control diet and a diet containing only $2.5 \%$ of control levels of BCAAs (BCAA-low). Food intake normalized to body weight and preference ratio for the low-BCAA diet were measured over 6 days of leptin infusion. (A) Leptin infusion reduced food intake in both genotypes such that there was an overall treatment effect, but no genotype effect or treatment by genotype interaction. ${ }^{*} P<0 \cdot 05$ compared to respective saline-infused group. ${ }^{\sharp} P<0 \cdot 05$ for individual diet choices compared with saline control. (B) KO mice exhibited a marked shift in preference toward the low-BCAA diet, and this shift was unaffected by leptin treatment. ${ }^{*} P<0 \cdot 05$ compared to WT-control.

doses of leptin, resulting in a physiological normalization of circulating leptin levels in the low dose, and a physiological increase with the high dose.

Normalization of circulating leptin had several affects in the $\mathrm{KO}$ mice, including reduced food intake and body adiposity. Interestingly, the $\mathrm{KO}$ mice exhibited a much larger reduction in body adiposity in response to leptin compared with WT mice. Since the KO mice are lean with reduced circulating leptin, it would be logical to speculate that they are more sensitive to exogenous leptin. However, leptin produced a relatively similar decrease in food intake in both genotypes. As such, it is unclear why $\mathrm{KO}$ mice responded to leptin with a larger reduction in adiposity, and this difference could be due to direct effects of increased BCAAs on the adipocyte or to the metabolic consequences of increased BCAAs or deletion of BCATm. Regardless, these data clearly indicate that the doses of leptin infused into the $\mathrm{KO}$ mice were physiologically relevant.
While the KO mice clearly responded to leptin, neither dose of leptin had any effect to reduce Npy or Agrp expression in these mice. To confirm that leptin is capable of reducing Npy and Agrp in these mice, we injected a very high-dose leptin into fed and fasted BCATm-KO mice. At these pharmacological doses, leptin blocked the fasting-induced increase in Agrp and Npy. Thus, we conclude that BCATm$\mathrm{KO}$ mice are capable of responding to leptin, but that low leptin levels are not responsible for the observed increase in Npy and Agrp expression. Instead, these data are more consistent with innate alterations in brain BCAA sensing or metabolism as contributing to the alterations in hypothalamic neuropeptide gene expression.

While BCATm-KO mice exhibit leptin-independent increases in Npy and Agrp, absolute food intake in these mice was only modestly altered. Previous work in these mice demonstrated that $\mathrm{KO}$ mice were smaller and leaner than controls, but that they consumed more food when that intake was normalized to body weight (She et al. 2007). In our hands, BCATm-KO mice are consistently smaller and leaner than controls, and initial work in Experiment 2 also indicated that they consumed similar amounts of food on an absolute basis and thus more food on a per gram body weight basis. However, subsequent experiments did not detect this difference, with $\mathrm{KO}$ mice showing a slight decrease in absolute food intake, but no change when intake was normalized to their reduced body weight. Taken together, we conclude that absolute food intake is relatively unperturbed in these mice, despite the markedly increased BCAAs. Thus, these data suggest that either increased BCAAs have no effect on food intake or some other defect (loss of glial BCAA metabolism) attenuates the response to increased BCAAs.

In contrast to their relatively normal absolute food intake, BCATm-KO mice exhibit a specific change in their preference for BCAAs. When given the choice between a control diet and a diet that was practically devoid of BCAAs, the low-BCAA diet constituted only $27 \%$ of the WT intake, but $62 \%$ the $\mathrm{KO}$ intake. Thus, the $\mathrm{KO}$ selected significantly more of the low-BCAA diet, suggesting that the
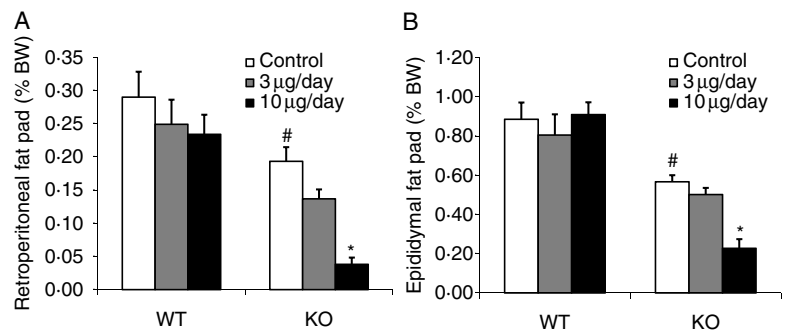

Figure 7 Fat pad weights in leptin-treated, wild-type (WT) and BCATm-KO mice. Retroperitoneal (A) and epididymal (B) fat pads were collected and weighed after 6 days of leptin infusion. Fat pads in BCATm-KO mice were reduced compared with WT, while leptin infusion further reduced fat pad weight in $\mathrm{KO}$ mice but not in WT mice. ${ }^{*} P<0 \cdot 05$ compared to respective saline-infused group; ${ }^{\sharp} P<0.05$ for WT-control vs KO-control. 


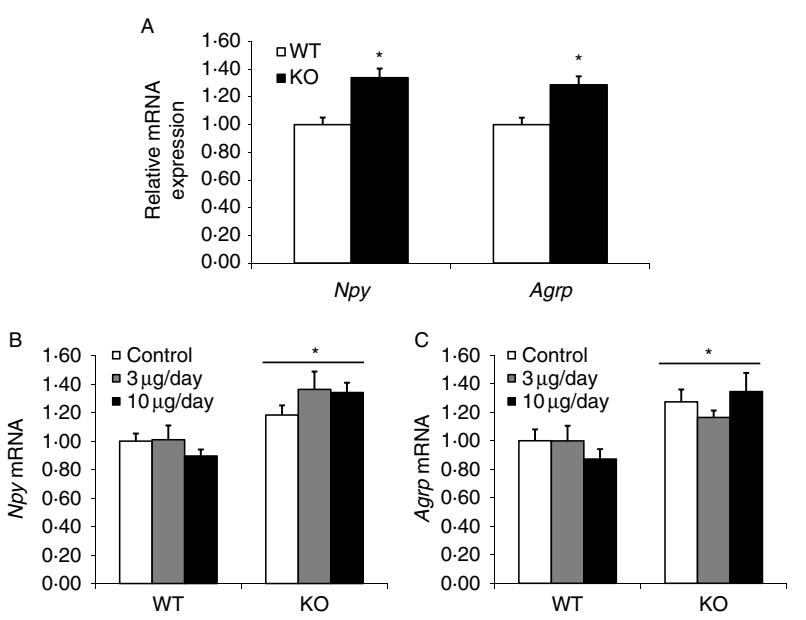

Figure 8 Hypothalamic Npy and Agrp gene expression in BCATm$\mathrm{KO}$ mice infused with leptin. Following 6 days of s.c. leptin infusion, hypothalamus was removed, total RNA was extracted, and Npy and Agrp expression was measured via real-time PCR. (A) Main effect of genotype on mRNA expression, demonstrating that $\mathrm{KO}$ mice have increased $N p y$ and Agrp $\left({ }^{*} P<0 \cdot 05\right)$. (B) Npy expression following leptin treatment. Leptin replacement has no effect on Npy expression. ${ }^{*} P<0 \cdot 05$ compared to WT-control. (C) Agrp expression following leptin treatment. Leptin replacement has no effect on Agrp expression. ${ }^{*} P<0 \cdot 05$ compared to WT-control.

BCATm-KO mice detect their abnormally high BCAAs and avoid consuming these amino acids. Surprisingly, this altered preference did not translate to protein in general. Thus, despite the evidence that leucine or BCAAs may signal excess protein, the $\mathrm{KO}$ mice chose normal amounts of protein when given the choice between low or high in protein. Because the low-BCAA and control diets were isocaloric and contained normal amounts of all amino acids besides BCAAs, BCATm mice had the luxury of avoiding BCAAs without negatively impacting energy or protein intake. However, when maintained on the standard LP and HP diets, avoiding BCAAs would result in a marked reduction in overall protein intake. Taken together, these data suggest that an elevation of BCAAs does not represent an overall signal of excess protein (Anderson et al. 1990) and instead suggests that the altered preference was limited to the three BCAAs. Although further work is required to define why $\mathrm{KO}$ mice avoid BCAAs, these data are consistent with previous work demonstrating that neuronal systems detect imbalances in dietary and/or circulating amino acids and alter food intake and selection to counteract these imbalances (Fromentin \& Nicolaidis 1996, Gietzen \& Rogers 2006, Rudell et al. 2011). In addition, this shift in preference was insensitive to leptin treatment, suggesting that the regulation of protein intake and selection is regulated separately from energy intake.

Finally, these data also highlight the possibility that glia may contribute to amino acid detection within the brain. Astrocytes are classically associated with neuronal support, functioning to maintain the extracellular environment by both regulating nutrient and metabolite balance and contributing to the uptake and break down of secreted neuropeptides (Volterra \& Meldolesi 2005). Yet, recent evidence indicates that astrocytes may also act in a regulatory role by detecting and secreting various signaling molecules, influencing the activity of nearby neurons, and having direct effects on synaptic plasticity (Araque et al. 1999, Newman 2003, Haydon \& Carmignoto 2006, Hermann et al. 2009). In particular, studies on brain glucose sensing have implicated astrocytes as a functional mediator of glucose detection (Young et al. 2000, Guillod-Maximin et al. 2004, Marty et al. 2005), while BCAA metabolism in astrocytes contributes to the glutamate-glutamine cycle and neuronal glutamate turnover (Yudkoff 1997, Lieth et al. 2001). Because BCATm-KO mice exhibit elevated circulating BCAAs but intact neuronal BCAA metabolism, we anticipated a phenotype more consistent with excess protein availability (i.e. reduced food intake and orexigenic neuropeptide expression). Instead, BCATm-KO mice exhibit a phenotype that is more representative of animals on LP diets. Therefore, while the current data do not provide unequivocal proof for the importance of glial BCAA metabolism in amino acid detection, these data together with previous work are consistent with the possibility that impaired glial BCAA metabolism may alter neuronal function and hypothalamic neuropeptide expression, and future experiments utilizing glial and neuronal specific knockouts will be required to directly test this hypothesis.

In summary, we have demonstrated that loss of BCAA transamination results in specific changes in diet selection and hypothalamic neuropeptide gene expression. These changes in neuropeptide expression and protein selection are independent of changes in circulating leptin, indicating not only that mechanisms other than leptin drive the increased Npy and Agrp but also that protein intake and selection may be unrelated to the regulation of energy intake. Finally, considering that hypothalamic Npy and Agrp expression is increased despite elevations in circulating amino acids, these data raise the possibility that impaired glial BCAA metabolism results in a loss of amino acid sensing within the brain.
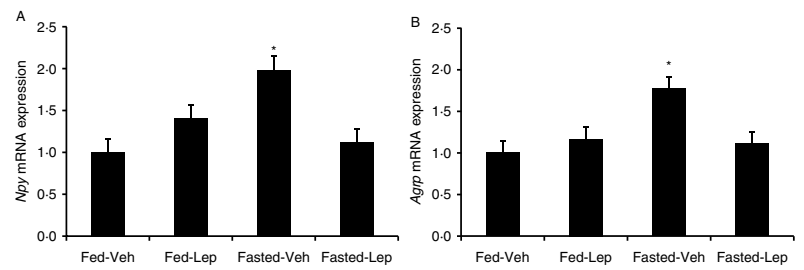

Figure 9 Effect of acute, high-dose leptin on Npy and Agrp mRNA. BCATm-KO mice were fed or fasted for $28 \mathrm{~h}$ and received i.p. injections of either leptin ( $5 \mu \mathrm{g} / \mathrm{g}$ body weight) or saline at the beginning of the fast (time 0), and then after 12 and $24 \mathrm{~h}$ (eight mice per group). Four hours after the final injection, hypothalamus was isolated to assess Npy and Agrp expression via real-time PCR. As expected, fasting significantly increased both $N p y(A)$ and Agrp (B). In both cases, leptin treatment blocked the fasting-induced increase in neuropeptide expression. ${ }^{*} P<0 \cdot 05$ compared fed-vehicle. 


\section{Declaration of interest}

The authors declare that there is no conflict of interest that could be perceived as prejudicing the impartiality of the research reported.

\section{Funding}

This work was supported by NIH Grants R01-DK081563 and P20-RR021945 to C D M, as well as core facilities supported in part by COBRE (NIH P20-RR021945) and NORC (NIH 1P30-DK072476) center grants from the National Institutes of Health.

\section{References}

Anderson GH \& Moore SE 2004 Dietary proteins in the regulation of food intake and body weight in humans. Journal of Nutrition 134 974S-979S.

Anderson SA, Tews JK \& Harper AE 1990 Dietary branched-chain amino acids and protein selection by rats. Journal of Nutrition 120 52-63.

Araque A, Parpura V, Sanzgiri RP \& Haydon PG 1999 Tripartite synapses: glia, the unacknowledged partner. Trends in Neurosciences 22 208-215. (doi:10.1016/S0166-2236(98)01349-6)

Baskin DG, Breininger JF \& Schwartz MW 1999 Leptin receptor mRNA identifies a subpopulation of neuropeptide $\mathrm{Y}$ neurons activated by fasting in rat hypothalamus. Diabetes 48 828-833. (doi:10.2337/diabetes.48.4.828)

Batterham RL, Heffron H, Kapoor S, Chivers JE, Chandarana K, Herzog H, Le Roux CW, Thomas EL, Bell JD \& Withers DJ 2006 Critical role for peptide YY in protein-mediated satiation and body-weight regulation. Cell Metabolism 4 223-233. (doi:10.1016/j.cmet.2006.08.001)

Blouet C \& Schwartz GJ 2010 Hypothalamic nutrient sensing in the control of energy homeostasis. Behavioural Brain Research 209 1-12. (doi:10.1016/j. bbr.2009.12.024)

Blouet C, Jo YH, Li X \& Schwartz GJ 2009 Mediobasal hypothalamic leucine sensing regulates food intake through activation of a hypothalamusbrainstem circuit. Journal of Neuroscience 29 8302-8311. (doi:10.1523/ jneurosci.1668-09.2009)

Brooks RC, Simpson SJ \& Raubenheimer D 2010 The price of protein: combining evolutionary and economic analysis to understand excessive energy consumption. Obesity Reviews 11 887-894. (doi:10.1111/j.1467789X.2010.00733.x)

Cota D, Proulx K, Smith KA, Kozma SC, Thomas G, Woods SC \& Seeley RJ 2006 Hypothalamic mTOR signaling regulates food intake. Science $\mathbf{3 1 2}$ 927-930. (doi:10.1126/science.1124147)

Du F, Higginbotham DA \& White BD 2000 Food intake, energy balance and serum leptin concentrations in rats fed low-protein diets. Journal of Nutrition 130 514-521.

Fromentin G \& Nicolaidis S 1996 Rebalancing essential amino acids intake by self-selection in the rat. British Journal of Nutrition 75 669-682. (doi:10. 1079/BJN19960172)

Gietzen DW \& Rogers QR 2006 Nutritional homeostasis and indispensable amino acid sensing: a new solution to an old puzzle. Trends in Neurosciences 29 91-99. (doi:10.1016/j.tins.2005.12.007)

Guillod-Maximin E, Lorsignol A, Alquier T \& Penicaud L 2004 Acute intracarotid glucose injection towards the brain induces specific $\mathrm{c}$-fos activation in hypothalamic nuclei: involvement of astrocytes in cerebral glucose-sensing in rats. Journal of Neuroendocrinology 16 464-471. (doi:10. 1111/j.1365-2826.2004.01185.x)

Hannah JS, Dubey AK \& Hansen BC 1990 Postingestional effects of a highprotein diet on the regulation of food intake in monkeys. American Journal of Clinical Nutrition 52 320-325.

Haydon PG \& Carmignoto G 2006 Astrocyte control of synaptic transmission and neurovascular coupling. Physiological Reviews 86 1009-1031. (doi:10. 1152/physrev.00049.2005)
Hermann GE, Van Meter MJ, Rood JC \& Rogers RC 2009 Proteinaseactivated receptors in the nucleus of the solitary tract: evidence for glialneural interactions in autonomic control of the stomach. Journal of Neuroscience 29 9292-9300. (doi:10.1523/JNEUROSCI.6063-08.2009)

Hutson SM, Wallin R \& Hall TR 1992 Identification of mitochondrial branched chain aminotransferase and its isoforms in rat tissues. Journal of Biological Chemistry 267 15681-15686.

Jean C, Rome S, Mathe V, Huneau JF, Aattouri N, Fromentin G, Achagiotis CL \& Tome D 2001 Metabolic evidence for adaptation to a high protein diet in rats. Journal of Nutrition 131 91-98.

Kanai Y, Segawa H, Miyamoto K, Uchino H, Takeda E \& Endou H 1998 Expression cloning and characterization of a transporter for large neutral amino acids activated by the heavy chain of 4F2 antigen (CD98). Journal of Biological Chemistry 273 23629-23632. (doi:10.1074/jbc.273.37.23629)

Korner J, Savontaus E, Chua SC Jr, Leibel RL \& Wardlaw SL 2001 Leptin regulation of Agrp and Npy mRNA in the rat hypothalamus. Journal of Neuroendocrinology 13 959-966. (doi:10.1046/j.1365-2826.2001.00716.x)

Lacroix M, Gaudichon C, Martin A, Morens C, Mathe V, Tome D \& Huneau JF 2004 A long-term high-protein diet markedly reduces adipose tissue without major side effects in Wistar male rats. American Journal of Physiology. Regulatory, Integrative and Comparative Physiology 287 R 934-R942. (doi:10. 1152/ajpregu.00100.2004)

Lieth E, LaNoue KF, Berkich DA, Xu B, Ratz M, Taylor C \& Hutson SM 2001 Nitrogen shuttling between neurons and glial cells during glutamate synthesis. Journal of Neurochemistry 76 1712-1723. (doi:10.1046/j.14714159.2001.00156.x)

Marty N, Dallaporta M, Foretz M, Emery M, Tarussio D, Bady I, Binnert C, Beermann F \& Thorens B 2005 Regulation of glucagon secretion by glucose transporter type 2 (glut2) and astrocyte-dependent glucose sensors. Journal of Clinical Investigation 115 3545-3553. (doi:10.1172/ JCI26309)

Mizuno TM \& Mobbs CV 1999 Hypothalamic agouti-related protein messenger ribonucleic acid is inhibited by leptin and stimulated by fasting. Endocrinology 140 814-817. (doi:10.1210/en.140.2.814)

Moran TH 2010 Hypothalamic nutrient sensing and energy balance. Forum of Nutrition 63 94-101.

Morrison CD, Morton GJ, Niswender KD, Gelling RW \& Schwartz MW 2005 Leptin inhibits hypothalamic Npy and Agrp gene expression via a mechanism that requires phosphatidylinositol 3-OH-kinase signaling. American Journal of Physiology. Endocrinology and Metabolism 289 E1051-E1057. (doi:10.1152/ajpendo.00094.2005)

Morrison CD, Xi X, White CL, Ye J \& Martin RJ 2007 Amino acids inhibit Agrp gene expression via an mTOR-dependent mechanism. American Journal of Physiology. Endocrinology and Metabolism 293 E165-E171. (doi:10. 1152/ajpendo.00675.2006)

Newgard CB, An J, Bain JR, Muehlbauer MJ, Stevens RD, Lien LF, Haqq AM, Shah SH, Arlotto M, Slentz CA et al. 2009 A branched-chain amino acid-related metabolic signature that differentiates obese and lean humans and contributes to insulin resistance. Cell Metabolism 9 311-326. (doi:10. 1016/j.cmet.2009.02.002)

Newman EA 2003 New roles for astrocytes: regulation of synaptic transmission. Trends in Neurosciences 26 536-542. (doi:10.1016/S01662236(03)00237-6)

Panksepp J \& Booth DA 1971 Decreased feeding after injections of aminoacids into the hypothalamus. Nature 233 341-342. (doi:10.1038/233341a0)

Peters JC \& Harper AE 1984 Influence of dietary protein level on protein selfselection and plasma and brain amino acid concentrations. Physiology and Behavior 33 783-790. (doi:10.1016/0031-9384(84)90048-9)

Ropelle ER, Pauli JR, Fernandes MF, Rocco SA, Marin RM, Morari J, Souza KK, Dias MM, Gomes-Marcondes MC, Gontijo JA et al. 2008 A central role for neuronal AMP-activated protein kinase (AMPK) and mammalian target of rapamycin (mTOR) in high-protein diet-induced weight loss. Diabetes 57 594-605. (doi:10.2337/db07-0573)

Rudell JB, Rechs AJ, Kelman TJ, Ross-Inta CM, Hao S \& Gietzen DW 2011 The anterior piriform cortex is sufficient for detecting depletion of an indispensable amino acid, showing independent cortical sensory function. Journal of Neuroscience 31 1583-1590. (doi:10.1523/JNEUROSCI.4934-10. 2011) 
Sandoval D, Cota D \& Seeley RJ 2008 The integrative role of CNS fuel-sensing mechanisms in energy balance and glucose regulation. Annual Review of Physiology 70 513-535. (doi:10.1146/annurev.physiol.70.120806.095256)

She P, Reid TM, Bronson SK, Vary TC, Hajnal A, Lynch CJ \& Hutson SM 2007 Disruption of BCATm in mice leads to increased energy expenditure associated with the activation of a futile protein turnover cycle. Cell Metabolism 6 181-194. (doi:10.1016/j.cmet.2007.08.003)

Smeets AJ, Soenen S, Luscombe-Marsh ND, Ueland O \& WesterterpPlantenga MS 2008 Energy expenditure, satiety, and plasma ghrelin, glucagon-like peptide 1, and peptide tyrosine-tyrosine concentrations following a single high-protein lunch. Journal of Nutrition 138 698-702.

Sorensen A, Mayntz D, Raubenheimer D \& Simpson SJ 2008 Protein-leverage in mice: the geometry of macronutrient balancing and consequences for fat deposition. Obesity 16 566-571. (doi:10.1038/oby.2007.58)

Sweatt AJ, Garcia-Espinosa MA, Wallin R \& Hutson SM 2004a Branched-chain amino acids and neurotransmitter metabolism: expression of cytosolic branched-chain aminotransferase (BCATc) in the cerebellum and hippocampus. Journal of Comparative Neurology 477 360-370. (doi:10.1002/cne.20200)

Sweatt AJ, Wood M, Suryawan A, Wallin R, Willingham MC \& Hutson SM $2004 \mathrm{~b}$ Branched-chain amino acid catabolism: unique segregation of pathway enzymes in organ systems and peripheral nerves. American Journal of Physiology. Endocrinology and Metabolism 286 E64-E76. (doi:10. 1152/ajpendo.00276.2003)

Tews JK, Kim YW \& Harper AE 1979 Induction of threonine imbalance by dispensable amino acids: relation to competition for amino acid transport into brain. Journal of Nutrition 109 304-315.

Tome D 2004 Protein, amino acids and the control of food intake. British Journal of Nutrition 92 (Suppl 1) S27-S30. (doi:10.1079/BJN20041138)

Volterra A \& Meldolesi J 2005 Astrocytes, from brain glue to communication elements: the revolution continues. Nature Reviews. Neuroscience 6 626-640. (doi:10.1038/nrn1722)

Westerterp-Plantenga MS 2003 The significance of protein in food intake and body weight regulation. Current Opinion in Clinical Nutrition and Metabolic Care 6 635-638. (doi:10.1097/00075197-200311000-00005)
Westerterp-Plantenga MS, Nieuwenhuizen A, Tome D, Soenen S \& Westerterp KR 2009 Dietary protein, weight loss, and weight maintenance. Annual Review of Nutrition 29 21-41. (doi:10.1146/annurev-nutr-080508141056)

White BD, He B, Dean RG \& Martin RJ 1994 Low protein diets increase neuropeptide $\mathrm{Y}$ gene expression in the basomedial hypothalamus of rats. Journal of Nutrition 124 1152-1160.

White CL, Whittington A, Barnes MJ, Wang Z, Bray GA \& Morrison CD 2009 HF diets increase hypothalamic PTP1B and induce leptin resistance through both leptin-dependent and -independent mechanisms. American Journal of Physiology. Endocrinology and Metabolism 296 E291-E299. (doi:10. 1152/ajpendo.90513.2008)

White CL, Purpera MN, Ballard K \& Morrison CD 2010 Decreased food intake following overfeeding involves leptin-dependent and leptinindependent mechanisms. Physiology and Behavior 100 408-416. (doi:10. 1016/j.physbeh.2010.04.006)

Wu G \& Knabe DA 1994 Free and protein-bound amino acids in sow's colostrum and milk. Journal of Nutrition 124 415-424.

Young JK, Baker JH \& Montes MI 2000 The brain response to 2-deoxy glucose is blocked by a glial drug. Pharmacology, Biochemistry, and Behavior 67 233-239. (doi:10.1016/S0091-3057(00)00315-4)

Yudkoff M 1997 Brain metabolism of branched-chain amino acids. Glia 21 92-98. (doi:10.1002/(SICI)1098-1136(199709)21:1<92::AIDGLIA10>3.0.CO;2-W)

Zhang Y, Guo K, LeBlanc RE, Loh D, Schwartz GJ \& Yu YH 2007 Increasing dietary leucine intake reduces diet-induced obesity and improves glucose and cholesterol metabolism in mice via multimechanisms. Diabetes $\mathbf{5 6}$ 1647-1654. (doi:10.2337/db07-0123)

\section{Received in final form 29 September 2011 \\ Accepted 3 October 2011 \\ Made available online as an Accepted Preprint 3 October 2011}

